\title{
Calidad en los servicios en los programas de postgrado de la Universidad del Zulia
}

\author{
Quality of services in the postgraduate programs of the University of Zulia
}

\begin{tabular}{lr}
\hline Calixto Jesús Hernández Gil & $\begin{array}{l}\text { Artículo recibido en junio } 2019 \\
\text { Arbitrado en julio } 2019\end{array}$ \\
calixtohg19@gmail.com & Publicado en septiembre 2019 \\
Código ORCID: 0000-0002-7945-7729 & \\
Universidad del Zulia, Venezuela &
\end{tabular}

\section{Resumen}

La investigación tuvo como objetivo analizar la calidad en los servicios en los programas de postgrado de la Universidad del Zulia. Metodológicamente fue de tipo analítica, correspondiéndole un diseño no experimental, transeccional y de campo. Se realizó tomando en consideración los programas de postgrado del área de las ciencias económicas y sociales de la Universidad del Zulia en sus tres Núcleos: Maracaibo, Costa Oriental del Lago y Punto Fijo, con la participación de 19 sujetos informantes. La recolección de información se llevó a cabo a través de la técnica de la encuesta, mediante la aplicación de un instrumento del tipo cuestionario, contentivo de 24 ítems. Los resultados alcanzados mostraron que la calidad en el servicio ostenta una alta presencia, ubicándola en una fortaleza para la gestión.

\begin{abstract}
The research aimed to analyze the quality of services in the graduate programs of the Universidad of Zulia. Methodologically it was of an analytical type, corresponding to a non-experimental, transectional and field design. It was carried out taking into consideration the postgraduate programs in the area of economic and social sciences of the University of Zulia in its three Nuclei: Maracaibo, East Coast of the Lake and Punto Fijo, with the participation of 19 informant subjects. The collection of information was carried out through the survey technique, through the application of a questionnaire-type instrument, containing 24 items. The results achieved show that the quality of the service has a high presence, placing it in a strength for management.
\end{abstract}

Palabras clave:

Calidad en los servicios prestados; manejo de reclamos; reputación en el mercado; respuesta oportuna de servicio, trato personal

\section{Keywords:}

Quality in the services provided; handling of claims; reputation in the market; timely response of service; personal treatment 
INTRODUCCIÓN

La nueva filosofía de gestión que persiguen las organizaciones, con la intención de perfeccionarse día a día haciendo uso de los recursos existentes, ha conllevado a describir conceptos como gestión del conocimiento, planeación estratégica, mejora continua, gestión de calidad, calidad del servicio, marketing, desarrollos tecnológicos, estrategias competitivas, entre otros.

Los mismos han venido a revolucionar no sólo la terminología de la administración actual, sino también, en el seno mismo de las organizaciones en cuanto a su cultura y metodologías de trabajo, orientadas incesantemente a parámetros como: reducción de costos, mejora de la calidad de productos y servicios, incrementar el mercado a través de la preferencia de los clientes con aplicación de estrategias efectivas de marketing, implementar elementos innovadores generadores de valor agregado; traduciéndose, en definitiva, en mayores ventajas competitivas.

La dinámica de los cambios actuales de la sociedad plantea, a las organizaciones en general, la necesidad de transformarse en función de nuevas demandas derivadas de los avances científicos, tecnológicos, humanísticos, así como también del contexto económico social. Se convierte entonces en un reto, para ellas, la búsqueda de soluciones que la adecuen permanentemente a estos cambios del mundo actual, unas adoptan modelos, otras filosofias de trabajo, en síntesis, todas intentan acercarse al mundo de la competitividad como alternativa de existencia.

En este contexto, en los últimos años los sistemas y modelos de gestión de la calidad del servicio han comenzado a tener una amplia aceptación en el ámbito socio-educativo. En especial, es en el ámbito universitario donde la adopción de los sistemas de calidad de servicio ha contado con una mayor tradición en estas últimas décadas. Por el contrario, únicamente desde hace pocos años se está produciendo la adopción de estos modelos de gestión de calidad del servicio en el sector de los postgrados en las universidades públicas.

Este hecho resulta especialmente visible en el caso de los servicios de postgrados en la Universidad del Zulia. En los últimos veinte años esta organización ha vivido un intenso progreso, pues de tratarse de unos centros de servicios catalogados con baja demanda por la sociedad, han ido evolucionando hasta lograr una gran demanda social. Unos centros educativos para los cuales se exige una gestión lo más eficaz y eficiente posible, con el objetivo de ofrecer la mejor asistencia a los usuarios, de manera que satisfagan sus necesidades y expectativas en cuanto al servicio recibido.

En efecto, en la situación actual de crisis económica, las universidades públicas se enfrentan a un escenario de recorte de ingresos y de incremento de las necesidades sociales, especialmente en materia de educación posgraduada; por ello, la eficiencia en la gestión de la calidad de servicio, con el objeto de racionalizar costos, coordinar prestaciones del servicio y atender a las necesidades reales de los usuarios, resulta una necesidad imperiosa.

Partiendo de esta realidad, el sector de los estudios de postgrado donde hasta hace no mucho tiempo su gestión enfocada a la calidad del servicio estaba poco profesionalizada, se está introduciendo en una cultura de gestión la 
cual en el pasado reciente no existía. Resulta evidente entonces, cómo la política de impulso adoptada por las universidades, tanto públicas como privadas, ha tenido un impacto considerable.

En ese sentido, afirma Cobra (2001), es el usuario quien determina qué es la calidad de servicio, la cual está en función de unos requisitos que han de establecerse a fin de satisfacer sus necesidades, por lo cual no se logrará calidad en el servicio si no existe interacción entre las expectativas y los estándares de desempeño, combinadas con la percepción de los clientes. Por consecuente, la naturaleza de la competencia, específicamente en el ámbito de servicios, no sólo se fundamenta en tener una gran plataforma tecnológica, sino también en valerse de esfuerzos integrales dirigidos en gran parte a la satisfacción de las necesidades del usuario, a través de la excelencia en la calidad de los servicios ofrecidos.

Particularmente, en los servicios prestados por las instituciones universitarias, los principales flujos de comunicación e interacción cliente-empleados tienen lugar en el propio proceso de prestación del servicio; es decir, se materializa por canales directos entre la universidad y el usuario, entre el empleado interno productor de los servicios y los clientes externos quienes lo reciben. Por tal motivo, la calidad de servicio percibida genera una imagen de la institución, a través de una dimensión instrumental definida por los aspectos físicos del servicio, $y$ una funcional enmarcada dentro de los aspectos intangibles o psicológicos del desempeño del servicio.

Cabe destacar, los programas de postgrado de la Universidad del Zulia no escapan a esta realidad, por lo cual se requiere analizar cómo están gestionando la calidad de los servicios prestados, así se puedan detectar fortalezas y debilidades, con la finalidad de brindar información pertinente a los tomadores de decisión para desarrollar estrategias dirigidas a mejorar el nivel de satisfacción de sus clientes (docentes, estudiantes, empleados, obreros, proveedores).

En el caso específico de estudio, el objetivo a alcanzar fue: Analizar la calidad en los servicios en los programas de postgrado de la Universidad del Zulia, donde el investigador ha podido constatar, a través de la observación directa y de información obtenida de los usuarios, algunos aspectos que afectan su funcionamiento; lo cual repercute en la calidad del servicio prestado.

\section{Calidad en los servicios}

Los servicios de calidad, como lo expone Medianero (2006), son servicios confiables en el sentido de que desempeñan la función para la cual se diseñaron y la ejecutan bien. El impacto de la calidad de un servicio sobre la ventaja competitiva es doble. Primero, suministrar servicios de alta calidad genera una reputación de la organización, a su vez, esta reputación incrementada permite que la organización cobre un mayor precio por sus servicios.

El segundo impacto de la calidad del servicio prestado en la ventaja competitiva proviene de la mayor eficiencia, por consiguiente, menores costos unitarios originados por una mayor calidad del servicio. En este caso el mayor efecto lo constituye el impacto de la calidad en la productividad. Una mayor calidad del servicio significa que se pierde menos tiempo por trabajador suministrando servicios fuera de lo normal, y se emplea menos tiempo corrigiendo errores. Esto se 
traduce en mayor productividad por trabajador y, necesariamente, menores costos. En consecuencia, la alta calidad del servicio no sólo permite a una organización establecer mayores precios, sino también, disminuir los costos.

En este marco de referencia, la calidad de los servicios prestados, constituye un nuevo sistema de gestión organizacional, en la medida que sus conceptos modifican radicalmente los elementos característicos del sistema de calidad tradicionalmente utilizado en los países de occidente. Entre ellos a juicio de Paz (2005), se encuentran:

- Los valores y las prioridades que orientan la gestión organizacional.

- Los planteamientos lógicos que prevalecen en la gestión organizacional.

- Las características de los principales procesos de gestión y decisión.

- Las técnicas y metodologías aplicadas.

- El clima entendido como el conjunto de las percepciones que las personas tienen sobre relaciones, política de personal, ambiente, entre otros.

Afirma el autor antes citado que el nuevo enfoque de calidad en los servicios requiere una renovación total de la mentalidad de las personas y por tanto, una nueva cultura organizacional; debido a que entre otros aspectos, se tiene que poner en práctica una gestión participativa y una revalorización del personal no aplicada en los modos de administración tradicional. Para la implantación de la calidad en los servicios no existe un modelo que pueda copiarse.

Ahora bien, para Gutiérrez (2005), la calidad en el servicio evidencia un servicio económico, útil y satisfactorio para el usuario. Se dice que un servicio es de calidad cuando satisface necesidades del usuario, en función de parámetros como: reputación en el mercado, seguridad que el servicio confiere al cliente; insumos utilizados, en cuanto a la fiabilidad o capacidad que tiene el servicio para cumplir las funciones especificadas, sin fallos y por un periodo determinado; y de respuesta oportuna, cuando el proveedor del servicio prestado responde en caso de fallo del servicio.

A juicio del investigador, es importante tener presente que la calidad del servicio abarca los conceptos de eficiencia (al especificar características y atributos apropiados en un servicio), y de eficacia (al requerir que se satisfagan las necesidades de los clientes). Se puede parafrasear entonces, que la calidad del servicio es la capacidad de la organización para agregar valor a los recursos que consume. Este valor agregado es el que diferencia a un servicio de los similares de la competencia y determina el precio que los usuarios estarán dispuestos a pagar por el servicio que se ofrece la empresa.

Si los clientes están dispuestos a pagar por este valor agregado más de lo que gasta la empresa, entonces tiene beneficios y es productiva. Los beneficios de una empresa, en consecuencia, dependerán de su capacidad para generar actividades que den valor agregado a sus servicios, y esto dependerá de muchos factores, incluyendo la calidad y cantidad de servicios que ofrece y los gastos en que incurra para hacerlo. Es importante hacer notar que una mejor calidad del servicio no siempre significa mayor costo.

A efectos de la investigación, para analizar la calidad en los servicios prestados presente en los programas de postgrado del área de las ciencias 
económicas y sociales ofrecidos por la Universidad del Zulia, se consideró pertinente el uso de los parámetros propuestos por Paz (2005) y Gutiérrez (2005), dado que serían los más acertados a efectos de la población bajo estudio, por ello esta dimensión se asume bajo el estudio de los siguientes indicadores: reputación en el mercado, insumos utilizados, respuesta oportuna, trato personal, manejo de los reclamos y la sensibilidad de los clientes a la calidad del servicio.

\section{Reputación en el mercado}

En este orden de ideas, para Gutiérrez (2005) la reputación de una organización de servicios en el mercado hace referencia a la percepción buena o mala que los usuarios pueden tener sobre la misma. La percepción sobre algo o alguien llega a través de imágenes, impresiones o sensaciones externas. La reputación de las organizaciones de servicios se encuentra influida de manera creciente, y significativa, por la reputación online, a partir de los climas de opinión generados por los usuarios en los diferentes contextos de sociabilidad online. También se ha definido, según Paz (2005) como: "La construcción social alrededor de la credibilidad, fiabilidad, moralidad y coherencia que se tiene de dicha organización" (p. 32).

Esto es, la reputación en el mercado sólo es parcialmente controlable ya que se crea y recrea a partir de percepciones que conforman un estado de opinión, consideración o valoración de otros, a partir de las decisiones, así como comportamientos ético-morales y profesionales de estas instituciones. Asimismo se puede sostener, a juicio del Equipo Vértice (2008), que la reputación en el mercado es una construcción social, un producto creado, acumulado de forma colectiva y que de manera inevitable tiene efectos positivos o negativos sobre el despliegue social, económico, entre otros, de la organización involucrada, al tener una evidente connotación pública.

Se puede inferir entonces, a criterio del investigador, que la gestión de la reputación en el mercado, por parte de las organizaciones de servicio, no hará referencia a cómo piensan en la forma de manipular la reputación, sino que se debe referir a las técnicas que la organización utilizará para entender a sus usuarios para ofrecerles un servicio a la altura de sus expectativas. Al respecto, la comunicación es uno de los componentes vitales de las organizaciones para lograr la proyección de su imagen pero es importante resaltar que lo que comunica la empresa deba tener coherencia con sus actitudes tanto en los ámbitos comerciales, sociales y económicos.

\section{Insumos utilizados}

Para Porter (2009), los insumos incluyen además de las materias primas todos los artículos y provisiones para el consumo, así como los activos maquinarias, equipos y edificios. Aunque los insumos se asocian generalmente con actividades primarias, están presentes en cada actividad de valor, incluyendo las actividades de apoyo. En general los insumos pierden sus propiedades y características para transformarse y formar parte del producto final. Para el caso de servicios, a los insumos se les considera como los recursos de entrada al proceso cuyo flujo de salida es el servicio entregado. 
En esta perspectiva, para Gutiérrez (2005) los insumos son bienes o servicios que son adquiridos por las empresas, y a su vez, son transformados en productos o servicio durante el proceso de producción de las mismas. Una definición más elaborada, dada por el autor, es que los insumos son bienes o servicios que se introducen y concentran en el proceso de producción de las empresas, y que, por medio de las actividades del personal de producción, apoyándose en las maquinarias y equipos, son convertidos en diferentes bienes o servicios, los cuales poseen un mayor valor agregado.

En este orden de ideas, a criterio del investigador, un insumo es aquel bien que es objeto de consumo en el proceso de producción de otro bien. En ciertas circunstancias esta expresión hace equivalencia al de materia prima, cuya mayor utilización se puede presentar en el área productiva. Normalmente, se denomina a los insumos como: factores de producción o recursos productivos. Frecuentemente los insumos cambian sus características o propiedades para convertirse y constituir parte del producto final. Cuando se hace referencia a los servicios, se establece que los recursos son aquellos que se introducen al proceso y cuya salida es el servicio entregado, entre ellos la calidad del servicio se convierte en insumo.

\section{Respuesta oportuna de los servicios}

En esta línea, para Grande (2005) los servicios por su intangibilidad no pueden producirse por adelantado a la demanda del cliente, pero deben entregarse al cliente al momento de la demanda o de forma posterior. Esto significa que por 1o general, las operaciones de servicios deben planear niveles de producción que se acerquen o igualen al momento en que se presenta la necesidad del cliente.

Ahora bien, la respuesta oportuna como atributo de calidad del servicio prestado, se refiere a la actitud que muestra la organización para ayudar a los clientes y para suministrar el servicio a la brevedad de tiempo posible; también es considerado parte de este punto el cumplimiento de los compromisos contraídos, así como lo accesible que puede ser la organización para el cliente, es decir, las posibilidades de entrar en contacto con la misma y la factibilidad con que pueda lograrlo. Por su parte, Gutiérrez (2010) refiere que, aun cuando existen pautas para desempeñar correctamente el servicio y tratar bien a los clientes, no es seguro que se brinde un servicio de alta calidad y el tiempo específico.

De lo antes expuesto, el investigador comprende que, la respuesta oportuna está referida a la disposición de ayudar a los clientes y proveerlos de un servicio rápido $\mathrm{y}$ oportuno. Los clientes no tienen por qué rogar para ser atendidos, ni para que sus dificultades o problemas sean solucionados, se debe estar al tanto de las dificultades, para ir un paso adelante de ellas y una buena forma de hacerlo es retroalimentándose con las observaciones de los clientes.

\section{Trato personal}

Los clientes de hoy día ya no solo buscan productos o servicios de alta calidad y bajos precios, sino también que se les brinde una buena atención, que se les trate con amabilidad, que se les haga sentir valorados e importantes. Un trato amable puede ser motivo suficiente para que un usuario elija a la organización 
antes de la competencia, mientras que un trato tosco o indiferente puede ser motivo suficiente para que se desanime en comprar o para que deje de hacerlo por más años que tenga siendo cliente de dicha organización.

En esta perspectiva para Riveros (2007), el servicio personalizado es la clave para un servicio de calidad. Los servicios personalizados han sabido conquistar a los usuarios y entablar relaciones a largo plazo. Al tratarse de un servicio hecho a la medida del cliente, significará más tiempo y dedicación, así como el contacto permanente para conocer sus requerimientos y saber qué esperan del servicio ofrecido.

A manera de resumen, a criterio del investigador, el factor que más influye en la capacidad para desarrollar relaciones de negocios, productivas y duraderas, no es el nivel educativo que los empleados hayan alcanzado, o que tan bien se expresen, o que tanto conozcan el producto, sino la calidad del trato que se les dé a los clientes. Este trato incluye: buenos modales, puntualidad, forma de vestir, manera de solucionar problemas, forma de hablar y dirigirse a los demás, y muchos otros aspectos que hacen que otros quieran tratar y hacer negocios con la organización o prefieran evitarla.

\section{Manejo de los reclamos}

En todo negocio siempre existirán las quejas o reclamos por parte del cliente, ya sea que se trate de un cliente muy exigente, o sea la organización la que haya cometido algún error, por ejemplo, haber brindado un mal servicio o una mala atención. A juicio de Fontanez (2006) cada vez que surjan estos problemas, una regla general en el manejo de las quejas y reclamos, es mantener la calma en todo momento. Siempre se debe mantener una actitud serena y por ningún motivo discutir con el cliente.

En este orden de ideas, según Jareño (2009), cuando un cliente plantea un conflicto, es importante la forma en que se le trata. Eso puede fortalecer la relación con el cliente, o darle el impulso final para que decida cambiar de empresa. El primer paso es evitar la confrontación (ya que no importa quién tiene razón), escucharlo y buscar una solución satisfactoria, tanto para él como para la empresa. Así, se logrará retenerlo. El segundo paso para construir clientes fieles y fanáticos, según el autor citado, se relaciona con la resolución de reclamos y el tratamiento de las situaciones conflictivas.

En virtud de lo antes expuesto, el investigador comprende que, existen dos puntos de vista muy extendidos respectos a las quejas o reclamos: el más común es que las quejas constituyen una "enfermedad" que debe ser evitada a toda costa. Las personas y empresas que mantienen este punto de vista creen que "nunca se equivocan", nunca comenten errores y si algo ocurre accidentalmente, como no debería ser, no es culpa de ellos. El otro punto de vista es cuando la queja representa una "oportunidad de oro" si es manejada con efectividad, para mantener, y en algunos casos aumentar, los niveles de lealtad de los clientes, además, durante el proceso de solución de la queja o reclamo la organización tendrá la oportunidad de aprender y solucionar los aspectos para que la situación no se repita, y tenga cada vez más éxito como organización. 


\section{Sensibilidad de los clientes a la calidad del servicio}

En la mayoría de los casos los clientes perciben poco o en algunos casos no perciben la calidad de los servicios prestados, sin embargo, en ocasiones, es posible influir en los clientes potenciales y actuales para que la apreciación hacia los niveles de calidad esté en ascenso. Ahora bien, según Riveros (2007), para medir la sensibilidad de los clientes a la calidad del servicio, basta con considerar la insatisfacción del cliente, la cual se hace notar por diferentes vías. Algunas formales, otras informales. Es claro que un cliente expresa su reclamo cuando está insatisfecho, pero no todas las organizaciones tienen la misma reacción ante situaciones de insatisfacción similares. Un libro de quejas, buzón de sugerencias, comentarios al empleado de atención al público, teléfono de atención al cliente, son maneras en las que puede expresar su insatisfacción.

Dentro de estas diferentes vías, afirma Riveros (2007), también se puede observar que hay distinta "intensidad" del reclamo. No es lo mismo un comentario al pasar que el cliente realiza al cajero del supermercado ("iQué larga se hizo la fila hoy...!), que un reclamo volcado en el libro de quejas con evidencias de gran inconformidad. El cliente que llega al libro de quejas es porque otras vías le han fallado, o bien su molestia ha escalado a un nivel de envergadura.

Ante este panorama, a juicio del investigador, para las instituciones prestadoras de servicio es fundamental la interpretación de las necesidades de los clientes y la evaluación del desempeño satisfactorio de los servicios prestados, lo que refuerza la competitividad y establece una relación con el cliente. Por ello medir la sensibilidad de los clientes a la calidad del servicio debe ser prioridad para este tipo de organizaciones.

En la prestación de sus servicios, la empresa obtiene grandes oportunidades para la obtención de ventajas competitivas de largo plazo, siendo que, estas ventajas pueden estar relacionadas a la sensibilidad de los clientes de la calidad del servicio prestado y a su proceso de provisión. Así, la calidad en servicios es percibida por los clientes, y lo que cuenta es la forma como ella es percibida, medida por la sensibilidad de estos.

\section{MÉTODO}

E1 objetivo de esta investigación consistió en analizar la calidad en los servicios en los programas de postgrado de la Universidad del Zulia, a través del análisis de los datos recolectados y los fenómenos observados en su propio medio para lograr resultados confiables, obtenidos por la utilización de los procedimientos metodológicos a ser aplicados, sin alterar la realidad a estudiar. Esta investigación se consideró de tipo analítica. De acuerdo al objeto de estudio, y la manera práctica de responder al objetivo, el diseño se enmarcó como no experimental, transeccional y de campo.

Para ello, el universo estuvo constituido por los programas de postgrados de la Universidad del Zulia. Mientras, para la población se trabajó con los programas de postgrados del área de las ciencias económicas y sociales ofrecidos por esta institución. Se destaca, el número total de programas de postgrados en el área reseñada se administra en los tres Núcleos de esta institución: Maracaibo, Costa Oriental del 
Lago y Punto Fijo; específicamente en el espacio geográfico comprendido por los estados Zulia y Falcón. Ahora bien, las unidades informantes, a quienes se les aplicó el instrumento para la obtención de la información, estuvieron compuestas por los Directores o Coordinadores de postgrado e investigación así como los Coordinadores de los programas de postgrado, pretendiendo cubrir el nivel gerencial, quedando constituidos por 19 sujetos informantes.

En concordancia con lo planteado, se utilizó la técnica de la encuesta a fin de obtener información referida al objetivo propuesto, utilizando como instrumento para la recolección de información el cuestionario, diseñado con preguntas cerradas, conformado por un número apropiado de 24 ítems, codificados con cinco alternativas de respuestas, representada de la siguiente manera: Siempre (S), Casi Siempre (CS), Algunas
Veces (AV), Casi Nunca (CN) y Nunca (N), elaborados por el investigador de acuerdo a las especificaciones establecidas en el objetivo.

Asimismo, la validez del instrumento se sometió a consulta a un jurado compuesto de seis (6) expertos, cuatro (4) en el campo de la calidad de los servicio para el aval del contenido, y dos (2) expertos metodológicos para avalar la construcción. Para el cálculo de la confiabilidad del instrumento se utilizó el coeficiente Alpha de Cronbach, a una prueba piloto 9 personas, alcanzando un coeficiente de 0.987.

Bajo esta perspectiva, para procesar los resultados de la aplicación del cuestionario se recurrió al método de la estadística descriptiva, el análisis se realizó con base a las medias aritméticas. Para ello, el investigador diseñó un (1) baremo, para el análisis de la media de los ítems, tal como se muestra en la Tabla 1.

Tabla 1. Baremo para la interpretación de la media aritmética Ítems

\begin{tabular}{lll}
\hline \multicolumn{1}{c}{ RANGO } & \multicolumn{1}{c}{ CATEGORÍA } & \multicolumn{1}{c}{ DESCRIPCIÓN } \\
$4,21-5$ & Muy alta presencia & $\begin{array}{l}\text { Si la presencia es muy alta o alta se considera una } \\
\text { fortaleza para las instituciones estudiadas, en cuanto a su } \\
\text { gestión de calidad del servicio }\end{array}$ \\
$3,41-4,20$ & Alta presencia & Si la presencia es moderada, baja o muy baja se considera \\
$2,61-3,40$ & Moderada presencia & una debilidad para las instituciones estudiadas, en cuanto \\
$1,81-2,60$ & Baja presencia & a su gestión de calidad del servicio \\
$1-1,80$ & Muy baja presencia &
\end{tabular}

Fuente: Elaboración propia.

\section{RESULTADOS}

El estudio se desarrolló utilizando la estadística descriptiva, analizando grupos de datos, siendo representados en forma de valores promedios en tablas de interés obtenidas a partir de la aplicación del instrumento, dirigido a un total de 19 informantes de los programas de postgrados mencionados, a continuación se presentan los elementos que conforman el evento de estudio, calidad en los servicios prestados.

\section{Reputación en el mercado}

En la Tabla 2, se muestra los resultados para la reputación en el mercado, con una media de 3.79, calificándolo con alta presencia en la gestión de la calidad del servicio de los programas de postgrado estudiados, 
delineándolo como fortalezas de la gestión. Al detalle, cada uno de los ítems analizados ostenta alta presencia, siendo considerados fortalezas de la gestión en cuanto a que se tienen definidos cuáles son los atributos del servicio que se presta. (3.95), asimismo, los servicios prestados son considerados por los usuarios como superiores a las demás alternativas existentes en el mercado (3.74), por lo general, se aplican técnicas para entender a sus usuarios ofreciéndoles un servicio a la altura de sus expectativas (3.63) y del mismo modo, La imagen que proyecta el servicio tiene coherencia con las actitudes de los proveedores del mismo (3.84).

Tabla 2. Reputación en el mercado

\begin{tabular}{lcc}
\hline \multicolumn{1}{c}{ Ítem } & Media & Categoría \\
\hline $\begin{array}{l}\text { 1. Se tienen definidos cuáles son los atributos del } \\
\text { servicio que se presta. }\end{array}$ & 3.95 & Alta presencia/Fortaleza \\
$\begin{array}{l}\text { 2. Los servicios prestados son considerados por los } \\
\text { usuarios como superiores a las demás alternativas }\end{array}$ & 3.74 & Alta presencia/Fortaleza \\
$\begin{array}{l}\text { existentes en el mercado. } \\
\text { 3. Se aplican técnicas para entender a sus usuarios } \\
\text { ofreciéndoles un servicio a la altura de sus } \\
\text { expectativas. }\end{array}$ & 3.63 & Alta presencia/Fortaleza \\
$\begin{array}{l}\text { 4. La imagen que proyecta el servicio tiene coherencia } \\
\text { con las actitudes de los proveedores del mismo. }\end{array}$ & 3.84 & Alta presencia/Fortaleza \\
Indicador & $\mathbf{3 . 7 9}$ & Alta presencia/Fortaleza \\
\hline
\end{tabular}

Fuente: Elaboración propia.

En consecuencia, los resultados alcanzados para el indicador concuerdan altamente con lo expresado por el Equipo Vértice (2008), quienes refieren que la reputación en el mercado es una construcción social, un producto creado, acumulado de forma colectiva y que de manera inevitable tiene efectos positivos o negativos sobre el despliegue social, económico, entre otros, de los programas de postgrado analizados de la Universidad del Zulia, al tener una evidente connotación y reconocimiento del dominio público.

\section{Insumos utilizados}

Como se observa en la Tabla 3, relacionada con el indicador insumos utilizados la media arrojada fue de 3.92 posicionándolo con una alta presencia en la gestión de la calidad del servicio de los programas de postgrados estudiados, siendo esto una fortaleza para la gestión. En específico, esto se debe a la alta presencia de la calidad en los insumos utilizados en la prestación de los servicios (3.95), se valora la calidad de los insumos utilizados en la prestación del servicio sobre otros atributos tales como precio, marca (3.95), asimismo, cuentan con las características especificadas en los protocolos del servicio (3.84) y por otro lado, la calidad del servicio se considera un insumo en la prestación del mismo (3.95). 
Tabla 3. Insumos utilizados

\begin{tabular}{|c|c|c|}
\hline Ítem & Media & Categoría \\
\hline $\begin{array}{l}\text { 5. Los insumos utilizados en la prestación de los } \\
\text { servicios son de calidad. }\end{array}$ & 3.95 & Alta presencia/Fortaleza \\
\hline $\begin{array}{l}\text { 6. Valoran la calidad de los insumos utilizados en } \\
\text { la prestación del servicio sobre otros atributos } \\
\text { tales como precio, marca. }\end{array}$ & 3.95 & Alta presencia/Fortaleza \\
\hline $\begin{array}{l}\text { 7. Los insumos utilizados cuentan con las } \\
\text { características especificadas en los protocolos del } \\
\text { servicio. }\end{array}$ & 3.84 & Alta presencia/Fortaleza \\
\hline $\begin{array}{l}\text { 8. La calidad del servicio se considera un insumo } \\
\text { en la prestación del mismo }\end{array}$ & 3.95 & Alta presencia/Fortaleza \\
\hline Indicador & 3.92 & Alta presencia/Fortaleza \\
\hline
\end{tabular}

Fuente: Elaboración propia.

En atención, lo antes expuesto coincide altamente con lo planteado por Gutiérrez (2005), para quien los insumos son bienes o servicios que son adquiridos por los programas de postgrado analizados de la Universidad del Zulia, y a su vez, son transformados en productos o servicio durante el proceso académico administrativo de los mismos. Por otro lado, para Porter (2009), en el caso de servicios, los insumos se les consideran como los recursos de entrada al proceso cuyo flujo de salida es el servicio entregado. A juicio del investigador, los programas de postgrado analizados deben estar claros cuáles son los insumos utilizados por ellos para la presentación del servicio, de tal manera, que los usuarios perciban la claridad de estos.

\section{Respuesta oportuna de los servicios}

Como se evidencia en la Tabla 4, el indicador arriba a la categoría de alta presencia con una media de 3.70, indicando una fortaleza para la gestión de la calidad del servicio en la población estudiada. En específico, se evidenció alta presencia en los ítems en cuanto a que: proporcionan la información requerida a los usuarios cuando ésta es solicitada $(4,11)$; asimismo, muestran disposición de ayudar a los clientes suministrando un servicio oportuno (3.90) y optimizan el tiempo de respuesta en el servicio de (3.42), siendo esto una fortaleza para la gestión. Por otra parte, se presenta una moderada presencia en relación a que algunas veces se tratan de conocer los niveles de satisfacción del usuario, para mejorar el tiempo de respuesta (3.37).

En este sentido, la alta presencia de los resultados coinciden con el planteamiento de Gutiérrez (2010), quien refiere que, aun cuando existen pautas para desempeñar correctamente el servicio y tratar bien a los usuarios, no es seguro que se brinde un servicio de alta calidad y el tiempo específico. A juicio del investigador, las autoridades que dirigen los programas de postgrado de la Universidad del Zulia, les corresponde hacer conocer a sus empleados que el tiempo de respuesta a los usuarios es un elemento primordial, el cual debe cuantificarse para conocer si se está siendo efectivo en el proceso académico - administrativo con el usuario. 
Tabla 4. Respuesta oportuna de los servicios

\begin{tabular}{lcc}
\hline \multicolumn{1}{c}{ Ítem } & Media & Categoría \\
\hline 9. Proporcionan la información requerida a los & 4.11 & Alta presencia/Fortaleza \\
$\begin{array}{l}\text { usuarios cuando ésta es solicitada. } \\
\text { 10. Muestra disposición de ayudar a los clientes } \\
\text { suministrando un servicio oportuno. }\end{array}$ & 3.90 & Alta presencia/Fortaleza \\
$\begin{array}{l}\text { 11. Optimizan el tiempo de respuesta en el } \\
\text { servicio de atención al cliente. }\end{array}$ & 3.42 & Alta presencia/Fortaleza \\
$\begin{array}{l}\text { 12. Tratan de conocer los niveles de satisfacción } \\
\text { del usuario, para mejorar el tiempo de } \\
\text { respuesta. }\end{array}$ & 3.37 & $\begin{array}{c}\text { Moderada } \\
\text { presencia/Debilidad }\end{array}$ \\
Indicador & $\mathbf{3 . 7 0}$ & Alta presencia/Fortaleza \\
\hline
\end{tabular}

Fuente: Elaboración propia.

\section{Trato personal}

Siguiendo con el análisis, en la Tabla 5 se presenta el detalle de los resultados correspondientes al indicador trato personal, la información muestra una alta presencia en la media alcanzada de 3.95, lo que implica una fortaleza para los programas de postgrado analizados en su gestión de la calidad del servicio. En específico, se presenta la alta presencia de los ítems en cuanto a que: se satisfacen las necesidades de los usuarios con un servicio al cliente enfocado en el buen trato humano (4.05), así como, mantienen contacto permanente con el usuario para conocer sus requerimientos, es decir, lo que esperan del servicio ofrecido (3.84) y procuran entregar el servicio a la hora estipulada (3.63). Por otra parte, se refleja una muy alta presencia al referirse los encuestados que la atención al usuario implica un excelente trato personal entre el proveedor del servicio y el usuario (4.26).

Estos resultados corroboran altamente lo planteado por Riveros (2007), quien refiere que el servicio personalizado es la clave para un servicio de calidad. Los servicios personalizados han sabido conquistar a los usuarios y entablar relaciones a largo plazo. Cuando los programas de postgrado analizados internalicen que un servicio hecho a la medida de los usuarios, significará más tiempo y dedicación, así como el contacto permanente para conocer sus requerimientos y saber qué esperan del servicio ofrecido, se lograra contar con usuarios satisfechos.

Tabla 5. Trato personal

\begin{tabular}{ccc}
\hline \multicolumn{1}{c}{ Ítem } & Media & \multicolumn{1}{c}{ Categoría } \\
\hline $\begin{array}{l}\text { 13. } \begin{array}{l}\text { Satisfacen las necesidades de los usuarios con un } \\
\text { servicio al cliente enfocado en el buen trato humano. }\end{array} \\
\begin{array}{l}\text { 14. La atención al usuario implica un excelente trato } \\
\text { personal entre el proveedor del servicio y el usuario. }\end{array}\end{array}$ & 4.26 & Alta presencia/Fortaleza \\
$\begin{array}{l}\text { 15. Mantienen contacto permanente con el usuario para } \\
\text { conocer sus requerimientos, es decir, lo que esperan } \\
\text { del servicio ofrecido. }\end{array}$ & 3.84 & Alta presencia/Fortaleza \\
$\begin{array}{l}\text { 16. Procuran entregar el servicio a la hora estipulada. } \\
\text { Indicador }\end{array}$ & 3.63 & Alta presencia/Fortaleza \\
\hline
\end{tabular}

Fuente: Elaboración propia. 


\section{Manejo de los reclamos}

En la Tabla 6 se muestra una media para el indicador manejo de los reclamos de 3.77, ubicándolo con alta presencia de la gestión de la calidad del servicio de los programas de postgrado estudiados, delineándolo como fortalezas de la gestión. Al detalle, se ostenta muy alta presencia en el ítem en cuanto a que: cuando un cliente plantea un conflicto se evita la confrontación, escuchándolo para buscar una solución satisfactoria (4.21). Por otra parte, se ostenta alta presencia para los encuestados cuando se consideran las quejas de los usuarios como una oportunidad para saber en qué se está fallando para tomar una acción correctiva (4.11) y una vez que ya se conocen las causas de los reclamos del usuario, se atacan para que no vuelvan a suceder (3.74) siendo considerados estos como una fortalezas de la gestión.

En cuanto, a que las quejas representan una molestia para el personal involucrado (3.00), este refleja una moderada presencia y por ende una debilidad de la gestión. A juicio del investigador, este en un indicativo de que los empleados de los programas de postgrado estudiados, algunas veces está preparado para la atención al usuario, así como para dar la respuestas a las solicitudes realizadas.

Este resultado de alta presencia en el indicador, valida lo expuesto por Jareño (2009), quien establece que cuando un usuario plantea un conflicto, es importante la forma como las autoridades de los programas de postgrado lo tratan. Eso puede fortalecer la relación con el usuario, o darle el impulso final para que decida cambiar de institución. Es por ello, que el primer paso es evitar la confrontación, escucharlo y buscar una solución satisfactoria, tanto para él como para la institución, y de esta manera, el programa logrará retenerlo. El segundo paso para construir clientes fieles $y$ fanáticos, se relaciona con la resolución de reclamos y el tratamiento de las situaciones conflictivas.

Tabla 6. Manejo de los reclamos

\begin{tabular}{|c|c|c|}
\hline Ítem & Media & Categoría \\
\hline $\begin{array}{l}\text { 17. Consideran las quejas de los usuarios como una } \\
\text { oportunidad para saber en qué se está fallando } \\
\text { para tomar una acción correctiva. }\end{array}$ & 4.11 & Alta presencia/Fortaleza \\
\hline $\begin{array}{l}\text { 18. Cuando un cliente plantea un conflicto se evita la } \\
\text { confrontación, escuchándolo para buscar una } \\
\text { solución satisfactoria. }\end{array}$ & 4.21 & Muy alta presencia/Fortaleza \\
\hline $\begin{array}{l}\text { 19. Las quejas representan una molestia para el } \\
\text { personal involucrado. }\end{array}$ & 3.00 & Moderada presencia/Debilidad \\
\hline $\begin{array}{l}\text { 20. Una vez que ya se conocen las causas de los } \\
\text { reclamos del usuario, se atacan para que no } \\
\text { vuelvan a suceder. }\end{array}$ & 3.74 & Alta presencia/Fortaleza \\
\hline Indicador & 3.77 & Alta presencia/Fortaleza \\
\hline
\end{tabular}

Fuente: Elaboración propia. 


\section{Sensibilidad de los clientes a la calidad del servicio}

La Tabla 7 describe el comportamiento del indicador sensibilidad de los clientes a la calidad del servicio con una media de 3.53 , reflejándose una alta presencia en la gestión de calidad del servicio de los programas encuestados, lo cual evidencia la fortaleza en la gestión.

Al detalle, se puede mostrar la alta presencia en los ítems en cuanto a que se emplean métodos para influir en los clientes (potenciales y actuales) para que la apreciación hacia los niveles de calidad esté en ascenso (3.47) y se considera que la lealtad de los clientes se cimienta sobre la capacidad de la institución de lograr en ellos una percepción de diferenciación en la experiencia de servicio (4.05), siendo ambos una fortaleza. Por otra parte, la moderada presencia en los ítems al referirse que algunas veces se da a conocer a los clientes los mecanismos de gestión de la calidad aplicados por la institución (3.21) y asimismo, se realizan investigaciones sobre la insatisfacción del cliente para medir la sensibilidad de los mismos a la calidad del servicio (3.37), reflejando una debilidad para la gestión y punto de mejora en la investigación realizada. El investigador coincide con estas debilidades; ya que, los programas de postgrados de la Universidad del Zulia no cuentan con los medios o herramientas para que el cliente refleje o dé a conocer su nivel de insatisfacción.

Tabla 7. Sensibilidad de los clientes a la calidad del servicio

\begin{tabular}{|c|c|c|}
\hline Ítem & Media & Categoría \\
\hline $\begin{array}{l}\text { 21. Emplean métodos para influir en los clientes } \\
\text { (potenciales y actuales) para que la apreciación } \\
\text { hacia los niveles de calidad esté en ascenso. }\end{array}$ & 3.47 & Alta presencia/Fortaleza \\
\hline $\begin{array}{l}\text { 22. Dan a conocer a los clientes los mecanismos de } \\
\text { gestión de la calidad aplicados por la institución. }\end{array}$ & 3.21 & Moderada presencia/Debilidad \\
\hline $\begin{array}{l}\text { 23. Realizan investigaciones sobre la insatisfacción } \\
\text { del cliente para medir la sensibilidad de los } \\
\text { mismos a la calidad del servicio. }\end{array}$ & 3.37 & Moderada presencia/Debilidad \\
\hline $\begin{array}{l}\text { 24. Consideran que la lealtad de los clientes se } \\
\text { cimienta sobre la capacidad de la institución de } \\
\text { lograr en ellos una percepción de diferenciación en } \\
\text { la experiencia de servicio. }\end{array}$ & 4.05 & Alta presencia/Fortaleza \\
\hline Indicador & 3.53 & Alta presencia/Fortaleza \\
\hline
\end{tabular}

Fuente: Elaboración propia.

En este sentido, la alta presencia de los resultados concuerdan con el planteamiento expresado por Riveros (2007), para quien medir la sensibilidad del usuarios de los programas de postgrado a la calidad del servicio, basta con considerar la insatisfacción del cliente, la cual se hace notar por diferentes vías. Es claro que un usuario expresa su reclamo cuando está insatisfecho, pero no todas las instituciones tienen la misma reacción ante situaciones de insatisfacción similares.

\section{Calidad en los servicios prestados}

Ahora bien, una vez presentados los hallazgos encontrados en los indicadores mencionados, se procede a exponer los resultados de la dimensión calidad en los servicios prestados en la Tabla 8 , en la cual se visualiza el comportamiento de la media de 3.78 indicando una alta 
presencia en la gestión de la calidad del servicio de los programa de postgrados de la Universidad del Zulia, ubicándola en una fortaleza para la gestión. En este sentido, se ostenta alta presencia en los indicadores tales como reputación en el mercado (3.79), insumos utilizados (3.92), respuesta oportuna de los servicios (3.70), trato personal (3.95), manejo de los reclamos (3.77) y la sensibilidad de los clientes a la calidad del servicio (3.53), posicionándose como una fortaleza de la gestión.

Visto así, estos resultados corroboran altamente lo planteado por Gutiérrez

Tabla 8. Calidad en los servicios prestados

\begin{tabular}{lcc}
\hline \multicolumn{1}{c}{ Indicadores } & Media & Categoría \\
\hline Reputación en el mercado & 3.79 & Alta presencia/Fortaleza \\
Insumos utilizados & 3.92 & Alta presencia/Fortaleza \\
Respuesta oportuna de los servicios & 3.70 & Alta presencia/Fortaleza \\
Trato personal & 3.95 & Alta presencia/Fortaleza \\
Manejo de los reclamos & 3.77 & Alta presencia/Fortaleza \\
Sensibilidad de los clientes a la calidad del servicio & 3.53 & Alta presencia/Fortaleza \\
Dimensión & $\mathbf{3 . 7 8}$ & Alta presencia/fortaleza \\
\hline
\end{tabular}

\section{CONCLUSIONES}

Con base en los hallazgos y resultados obtenidos en la investigación, que permitieron analizar la calidad en los servicios en los programas de postgrado de la Universidad del Zulia, se pudo conocer que la calidad en el servicio ostenta una alta presencia, ubicándola en una fortaleza para la gestión. En este sentido, existe una alta presencia en los indicadores tales como reputación en el mercado, insumos utilizados, respuesta oportuna de los servicios, trato personal, manejo de los reclamos y la sensibilidad de los clientes a la calidad del servicio.

De manera concreta, las debilidades encontradas en este objetivo se centran en
(2005), para quien la calidad en el servicio evidencia un servicio económico, útil y satisfactorio para el usuario. Se dice que un servicio es de calidad cuando satisface necesidades del usuario, en función de parámetros como: reputación en el mercado, seguridad que el servicio confiere al cliente; insumos utilizados, en cuanto a la fiabilidad o capacidad que tiene el servicio para cumplir las funciones especificadas, sin fallos y por un periodo determinado; y de respuesta oportuna, cuando el proveedor del servicio prestado responde en caso de fallo del servicio. los indicadores, respuesta oportuna de los servicios; ya que, algunas veces se tratan de conocer los niveles de satisfacción del usuario, para mejorar el tiempo de respuesta. En cuanto al manejo de los reclamos, se pudo conocer que algunas veces las quejas representan una molestia para el personal involucrado. Por otra parte, la sensibilidad de los clientes a la calidad del servicio cuando los encuestados consideraron que algunas veces se dan a conocer a los clientes los mecanismos de gestión de la calidad aplicados por la institución y que realizan investigaciones sobre la insatisfacción del cliente para medir la sensibilidad de los mismos a la calidad del servicio. 
REFERENCIAS

Cobra, M. (2001).Marketing de Servicios. 2da Edición. Editorial McGraw-Hill. Colombia

Equipo Vértice (2008). La calidad en el servicio del cliente. Editorial Vértice. Recuperado de https://books.google.co.ve/books?isbn $=8492533714$

Fontanez, D. (2006). Estrategias de servicio al cliente. Artículo en línea. Recuperado de http://www.gestiopolis.com/canales7 /mkt/estrategias-de-servicio-alcliente.htm

Grande, I. (2005). Marketing de servicios. 4ta Edición. Madrid: ESIC Editorial

Gutiérrez, C. (2005). Administración y Calidad. Octava reimpresión. México: Editorial Limusa. Grupo Noriega Editores S.A

Gutiérrez, H. (2010). Calidad total y productividad. 3ra edición. México: McGraw-Hill

Jareño, O (2009). Estrategias para mejorar su servicio de atención al cliente.
Artículo en línea. Recuperado de http://www.capsulasdemarketing.com /negocios/7-estrategias-para-mejorarsu-servicio-de-atencion-al-cliente.html

Medianero, D. (2006). Elaboración de planes estratégicos institucionales. Revista No. 16 de la Facultad de Ciencias Económicas de la Universidad de México. Volumen 17 Año V. México

Paz, R. (2005). Servicio al cliente: la comunicación y la calidad del servicio en la atención al cliente. Madrid: Editorial Ideas Propias

Porter, M. (2009). Ser competitivo. Edición actualizada y aumentada. Ediciones Pirámide, S.A., 2010. Barcelona: Ediciones Deustro

Riveros, P. (2007). Sistema de gestión de la calidad del servicio. Sea líder en mercados altamente competitivos. 3ra Edición. ECOE Ediciones. Bogotá, Colombia 